\title{
Assessment and Treatment of Challenging Behavior Maintained by a Nonvocal Mands Function
}

\author{
Sara Jeglum ${ }^{1,2} \cdot$ Jonathan D. Schmidt ${ }^{1,2} \cdot$ Morgan Hallgren $^{1} \cdot$ Jennifer Vetter ${ }^{1,2} \cdot$ Amanda Goetzel $^{1}$ \\ Published online: 15 September 2020 \\ (C) Association for Behavior Analysis International 2020
}

\begin{abstract}
Standard functional analysis procedures may require modifications to assess idiosyncratic variables, such as adult compliance with mands. In the literature, the mands function is largely represented by individuals who vocally communicate idiosyncratic requests. Although effective treatment procedures have been published, schedule thinning has rarely been conducted. Using a reversal design, a mands functional analysis was completed with a 12-year-old nonvocal male. Results showed differentiated rates of challenging behavior. Treatment consisted of differential reinforcement via a chained schedule with signaled availability. During schedule thinning, low rates of challenging behavior were maintained.
\end{abstract}

Keywords Adult compliance with mands $\cdot$ Problem behavior

Although the functional analysis (FA) is well established in identifying functions of challenging behavior for individuals with intellectual and developmental disabilities (Beavers, Iwata, \& Lerman, 2013), standard FA procedures may require modification to assess idiosyncratic variables, such as adult compliance with mands (Bowman, Fisher, Thompson, \& Piazza, 1997). In the literature, the mands function is largely represented by individuals with developmental disabilities who vocally communicate idiosyncratic requests through vocalizations (Schmidt et al., 2017). Mand content is widely variable and typically idiosyncratic (e.g., mands for rearrangement; Torres-Viso, Strohmeier, \& Zarcone, 2018; playing in a certain way; Bowman et al., 1997). However, there are limited functional analyses on nonvocal verbal behavior (Normand, Severtson, \& Beavers, 2008) and no study that formally examines an adult-compliance-with-nonvocal-mands function for challenging behavior. One study conducted by Eluri, Andrade, Trevino, and Mahmoud (2016) examined the challenging behavior of a participant who had complex vocal speech but rarely used spoken language to request; rather, he

Jonathan D. Schmidt

schmidtj@kennedykrieger.org

1 Department of Behavioral Psychology, Kennedy Krieger Institute, 707 N. Broadway, Baltimore, MD 21205, USA

2 Johns Hopkins University School of Medicine, Baltimore, MD, USA used gestures most reliably to mand. However, in the assessment phase, only vocal mands were honored, which limits the results of this study because the maintaining function was potentially not correctly identified.

The most common treatment components for challenging behavior maintained by adult compliance with mands include differential reinforcement of alternative behavior (DRA) via functional communication, DRA for compliance with adult mands, differential reinforcement of other behaviors (DRO), and extinction. Despite the literature demonstrating effective interventions, schedule thinning has rarely been used (Schmidt et al., 2017; Torres-Viso et al., 2018). The current case study demonstrates an application of assessment and treatment of challenging behavior maintained by access to adult compliance with nonvocal mands.

\section{Method}

\section{Participant and Setting}

Mike was a 12-year-old male diagnosed with Schaaf-Yang syndrome, autism spectrum disorder, stereotypic movement disorder with self-injurious behavior, anxiety disorder, depression, obsessive-compulsive disorder, and moderate intellectual disability. He was admitted to an inpatient hospital unit for the assessment and treatment of severe challenging behavior. Mike was nonvocal and communicated primarily with picture 
cards, gestures, signs, and one-syllable vocalizations. A speech-language pathologist completed a speech-language assessment using a modified version of the Rosetti InfantToddler Language Scale (Rossetti, 2006). His receptive language was estimated to be at the 27- to 30-month level, whereas his expressive language level was approximately $15-$ 21 months.

All sessions were $10 \mathrm{~min}$ in duration and conducted in living areas of the inpatient unit with other peers and staff present. Typically, 6 to 10 sessions were conducted each day, 5 days per week. A therapist and relevant session materials were present.

\section{Measurement and Interobserver Agreement}

Frequency data were collected on challenging behavior (i.e., emesis, head-banging, aggression, disruption, and biting others) and mands and displayed as responses per minute (RPM). Behaviors that were given programmed social consequences (described next) included emesis, aggression, and disruption; head-banging and biting others did not receive programmed social consequences and were blocked and ignored due to their severity.

Trained observers collected data on laptop computers using behavioral data program software (bDataPro; Bullock, Fisher, \& Hagopian, 2017). A second observer collected data for $25 \%$ of sessions across assessment and treatment. Sessions were divided into 10-s intervals. For frequency data, interobserver agreement (IOA) was calculated using the exact-intervalagreement method, in which an agreement was scored when both observers scored the same number and type of responses within an interval. IOA was $96 \%$ for all behaviors across assessment and treatment sessions.

\section{Experimental Design and Procedures}

\section{Functional Behavioral Assessment}

Indirect and Direct Assessments Trained behavior analysts conducted semistructured clinical interviews with Mike's parents and staff (modified from O'Neill et al., 2015), descriptive assessments, and observations during admission. Descriptive assessments included observing Mike in various activities throughout the day (e.g., activities of daily living, unit activities) and collecting information (e.g., antecedent, behavior, consequence data) about environmental contingencies. Overall, information suggested that adult noncompliance with mands, both typical and idiosyncratic, reliably evoked challenging behavior. These mands took several topographical forms, including one-syllable vocalizations (e.g., "buh"), signs (e.g., "please"), pointing to the requested item or activity, grabbing the requested item, and directing/leading the staff member.
Traditional social mands were defined as requests that most individuals emit on a regular basis and do not involve idiosyncratic features. Examples included going for a walk, sitting on a couch, and playing with toys (e.g., puzzles, matching, electronics). In addition to traditional social mands, Mike had a history of asking for reinforcers to be delivered in highly idiosyncratic ways. Idiosyncratic mands were defined as requests that are specific to an individual and do not result directly in the functional reinforcer; rather, these mands are precurrent to the reinforcer (Bowman et al., 1997; Owen et al., 2020). That is, the mand produces the behavior that leads to the reinforcer. Idiosyncratic mands included requests for others to wear clothing in specific ways, manipulate personal items (e.g., move around or interact with jewelry, identification cards, or clothing), provide specific forms of attention from certain people (e.g., dancing from one person and neck rubs from another person while sitting beside him), and take turns opening and closing cabinets repeatedly.

\section{FA of Challenging Behavior Maintained by Adult Compliance} with Mands Using a reversal design, an FA of challenging behavior maintained by adult compliance with mands (henceforth "mands FA") was completed. For both test and control conditions, a therapist, preferred toys, and the activities that Mike was likely to engage in mands in the presence of were available freely. Toys and activities identified through interviews with staff and observations from prior assessments included a handheld game console, gear toy, laminated puzzles, sitting on a couch with a therapist, and walking. Two minutes prior to a session, the therapist stated, "What would you like to do?" and honored all reasonable mands. Mands were occasionally unclear because there was no point-to-point correspondence with the functional reinforcer; in these situations, the therapist asked clarifying (e.g., "What do you mean by that?") or closed-ended ("Do you want [toy or activity]?") questions across conditions and/or allowed Mike to manipulate the therapist's body.

In the control condition, Mike directed the manner of play, and all reasonable mands were honored. All challenging behavior was ignored. In the test condition, the therapist directed the manner of play and stated, "It's time to play my way." The therapist directed Mike to play with toys differently than how he was playing with them (e.g., press other buttons on the handheld game console instead of the ones he was pressing) or with other toys (e.g., a toy kitchen or toy piano) in a different way. To more directly mimic interactions in the natural environment during play, there was no programmed number of directives per toy or activity and no predetermined criterion for changing the style of play to another toy, activity, or directive. All appropriate mands were ignored. Target challenging behaviors were reinforced by honoring the last mand emitted, along with all other reasonable mands for $30 \mathrm{~s}$. After $30 \mathrm{~s}$, the therapist again directed the manner of play. 


\section{Treatment}

Treatment was evaluated using a B-A-B design; sessions were $10 \mathrm{~min}$ in duration. Baseline procedures and contingencies were identical to the test condition of the mands FA. Treatment components consisted of signaled availability for DRA via functional communication, and DRO. Signaled availability was indicated using a visual board. The top half held a double-sided card with "Mike's Way" (discriminative stimulus $\left[\mathrm{S}^{\mathrm{D}}\right]$ ), indicating that his mands would be reinforced, and "Caregiver's Way" on the reverse side (stimulus delta $\left[\mathrm{S}^{\Delta}\right]$ ), indicating his mands would not be reinforced (Schmidt et al., 2017). On the bottom half, a green side and a red side signaled what items/activities were and were not available via picture cards depicting the same toys and activities from the mands FA.

The therapist used a chained schedule of reinforcement (Torres-Viso et al., 2018), in which Mike was required to not engage in challenging behavior during the Caregiver's Way (DRO) period to earn reinforcement (Mike's Way). At the outset of treatment, the DRA (Mike's Way) was implemented for the entire session $(10 \mathrm{~min})$. The reinforcement schedule was thinned systematically, generally after three consecutive sessions with challenging behavior rates below an $80 \%$ reduction from baseline. That is, the resetting DRO (Caregiver's Way) was initially set to $10 \mathrm{~s}$ and gradually increased with a terminal goal of $5 \mathrm{~min}$ for the DRO interval.

Caregiver's Way (DRO) The therapist flipped the top card to "Caregiver's Way," moved all picture cards to the red side, and stated, "It is time for Caregiver's Way. If you stay safe, we can play Mike's Way." The therapist directed Mike to play with toys differently than how he was playing or with other toys in a different way; three-step prompting was used for all adult directives. Contingent on challenging behavior, the DRO duration was extended by 1 min (Mike's Way correspondingly decreased by $1 \mathrm{~min}$ ), and the therapist stated, "Because you were unsafe, we'll spend more time in Caregiver's Way." If Mike emitted a mand during the DRO interval, the therapist stated, "Not right now. You may ask when it is Mike's Way." All subsequent mands were ignored.

Mike's Way (DRA) The therapist flipped the top card to "Mike's Way" and moved all picture cards (functional communication training was conducted prior to this evaluation) to the green side of the board. The therapist stated, "We can play Mike's Way now." Mike's mands were reinforced on a fixed-ratio 1 schedule (FR1) using picture cards or signs. Other less acceptable forms of communication (e.g., grabbing) were ignored. If Mike used a one-syllable vocalization ("buh") toward a therapist, the therapist prompted him to specify (e.g., "What do you mean by that?"). Challenging behavior was placed on extinction.

\section{Results}

\section{Mands FA}

Results (Fig. 1) depict elevated and differentiated rates of challenging behavior in the test ( $M=1.43 \mathrm{RPM})$ compared to the control $(M=0.004 \mathrm{RPM})$ condition. During the control condition, Mike frequently manded ( $M=1.51 \mathrm{RPM})$, directing play and activities with the therapist; when mands were reinforced contingent upon challenging behavior, mand rates were more elevated ( $M=2.85 \mathrm{RPM})$, likely because they were not reinforced on an FR1.

\section{Treatment}

In the first treatment phase, zero rates of challenging behavior were observed; mand rates were variable and occurred throughout Mike's Way ( $M=1.84$ RPM). During baseline, challenging behavior was elevated $(M=2.47 \mathrm{RPM})$, and mands consistently occurred ( $M=2.87$ RPM). After reintroducing treatment, there was a change in level, and low rates of challenging behavior were observed, remaining low throughout schedule thinning ( $M=0.12$ RPM). Mands continued to occur specifically during Mike's Way throughout schedule thinning $(M=1.73 \mathrm{RPM})$. Mand rates were differentiated between Mike's Way ( $M=1.13$ RPM) and Caregiver's Way ( $M=0.01 \mathrm{RPM})$, indicating stimulus control over when mands occurred (Fig. 2, bottom panel). Mand rates during Mike's Way periods $(M=2.06 \mathrm{RPM})$ were comparable to the mands FA ( $M=1.51$ RPM during the control condition; $M=2.85$ RPM in test). In the treatment evaluation, Mike rarely emitted mands during Caregiver's Way.

\section{Discussion}

This evaluation builds upon the prior literature by demonstrating how to assess and treat challenging behavior maintained by access to adult compliance with nonvocal mands. Favorable results were maintained even after schedule thinning. There has been limited research on challenging behavior maintained by adult compliance with mands, and none to our knowledge for individuals with nonvocal mands primarily expressed via pointing or gesturing. This is of particular importance because if adults interacting with the individual are not aware of what the individual is requesting due to it being idiosyncratic in nature or unclear in specificity, there is an increased risk of challenging behavior. It took time for staff to become familiar with what Mike was requesting by pointing and gesturing. Recognizing and understanding an individual's nonvocal mands are imperative prior to initiating assessment, and for some individuals with more limited repertoires of communication who also have highly idiosyncratic 
Emesis, Head-Banging, Aggression, Disruption, and Biting Others
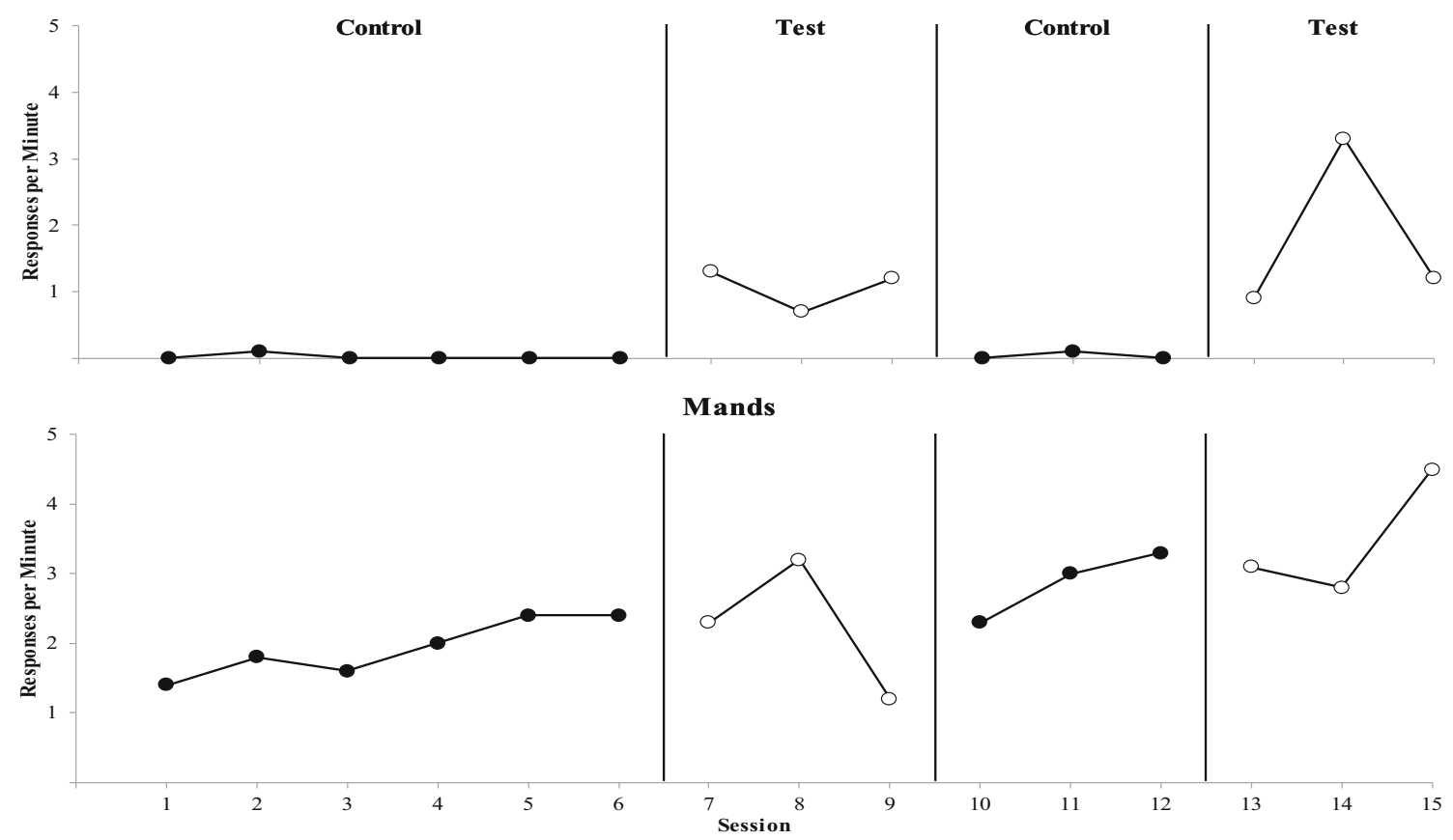

Fig. 1 . Mands FA. Closed circles represent control sessions, and open circles represent test sessions.

Emesis, Head-Banging, Aggression, Disruption, and Biting Others

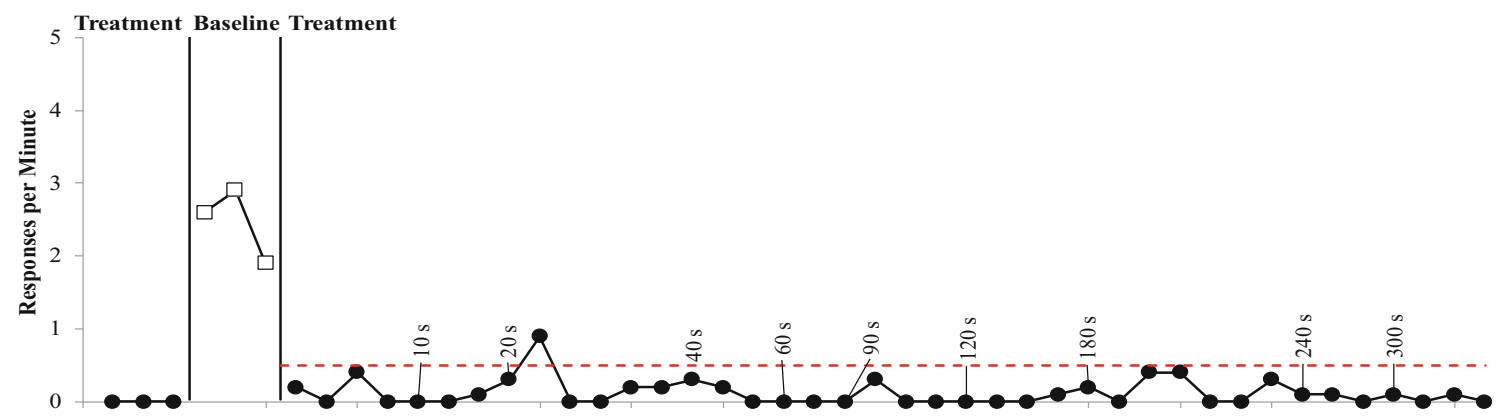

Mands

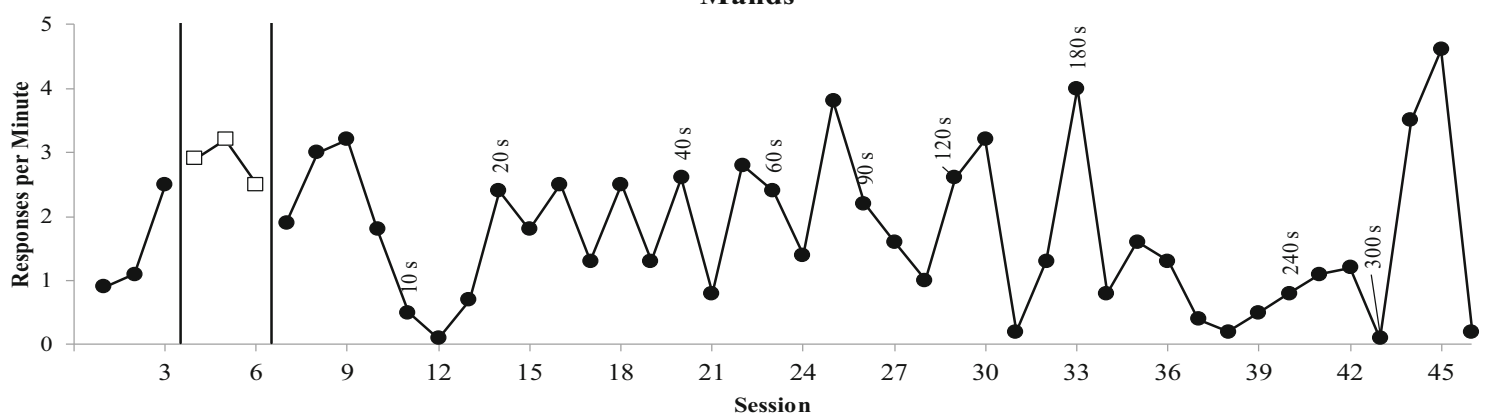

Fig. 2 Treatment evaluation. Closed circles represent treatment sessions, and open squares represent baseline sessions. In the bottom panel, open circles represent discriminative stimulus (SD) intervals, and closed circles represent stimulus delta intervals. During the first treatment phase and the beginning of the second treatment phase, SD intervals comprised the full 10 -minute session. 
requests, there may be an extensive time commitment for the practitioner to adequately delineate the specific contingencies. Additionally, this may impact procedural and treatment integrity; a limitation is we did not collect data on these two areas.

The rate of schedule thinning represents another limitation of our study. Given Mike's success as the Caregiver's Way period was extended, a terminal-probe approach could have been used to achieve terminal reduction goals quickly; however, previous assessments suggested Mike was sensitive to larger changes in contingencies. This is important to consider because larger changes in contingencies, including thinning of reinforcement and introducing novel people or settings, could greatly impact maintenance of challenging behavior and mand rates. In our study, a high level of stimulus control over when mands occurred was maintained throughout schedule thinning. It is possible that Mike's discrimination between when mands would and would not be honored may have been impacted (i.e., less exposure to the contingency) if terminal probes were used. All sessions were conducted in generalized settings (i.e., the living areas of the inpatient unit), rather than a private session room, which may have contributed to the success of schedule thinning and generalization and allowed for more opportunities for naturalistic mands (e.g., going for a walk).

Further replication of assessment and treatment with other individuals with challenging behavior maintained by nonvocal mands is warranted in naturalistic settings. In our study, only emesis, aggression, and disruption received programmed contingencies in the FA, limiting interpretation of the function of head-banging and biting others. Although functions were not confirmed for these two behaviors, it was essential for safety, and observations indicated these behaviors were in the same functional response class.

Future research could evaluate which intervention components were most effective in reducing and sustaining low rates of challenging behavior over time, specifically after generalization. For example, Mike frequently played with his siblings and exhibited the same patterns of challenging behavior when they would not comply with his mands. Thus, the social validity of these procedures in that context would be meaningful for his participation in family activities.

Motivating operations change frequently for individuals whose challenging behavior is maintained by an adultcompliance-with-mands function. When clinicians struggle to identify possible functions of challenging behavior related to idiosyncratic mands, it is crucial to consider how best to modify procedures to adequately discriminate between an adult-compliance-with-mands function and other functions. This study offers one set of procedures to address this issue in individuals with nonvocal communication.

\section{Compliance with Ethical Standards}

Conflict of Interest All of the authors declare that they have no conflict of interest.

Ethical Approval All procedures performed in this study involving human participants were in accordance with the ethical standards of the institutional and/or national research committee and with the 1964 Helsinki declaration and its later amendments or comparable ethical standards.

Informed Consent Informed consent was obtained from the caregivers of the participant included in the study. The data that support the findings of this study are available from the corresponding author upon reasonable request.

\section{References}

Beavers, G. A., Iwata, B. A., \& Lerman, D. C. (2013). Thirty years of research on the functional analysis of problem behavior. Journal of Applied Behavior Analysis, 46(1), 1-21. https://doi.org/10.1002/ jaba.30.

Bowman, L. G., Fisher, W. W., Thompson, R. H., \& Piazza, C. C. (1997). On the relation of mands and the function of destructive behavior. Journal of Applied Behavior Analysis, 30, 251-265.

Bullock, C. E., Fisher, W. W., \& Hagopian, L. P. (2017). Description and validation of a computerized behavioral data program: "bDataPro". The Behavior Analyst, 40(1), 275-285. https://doi.org/10.1007/ s40614-016-0079-0.

Eluri, Z., Andrade, I., Trevino, N., \& Mahmoud, E. (2016). Assessment and treatment of problem behavior maintained by mand compliance. Journal of Applied Behavior Analysis, 49, 383-387.

Normand, M. P., Severtson, E. S., \& Beavers, G. A. (2008). A functional analysis of non-vocal verbal behavior of a young child with autism. The Analysis of Verbal Behavior, 24, 63-67.

O’Connor, J. T., Sorenson-Burnworth, R. J., Rush, K. S., \& Eidman, S. L. (2003). A mand analysis and levels treatment in an outpatient clinic. Behavioral Interventions, 18, 139-150.

O’Neill, R. E., Horner, R. H., Albin, R. W., Sprague, J. R., Storey, K., \& Newton, J. S. (2015). Functional assessment and program development for problem behavior (3rd ed.). Stamford, CT: Cengage Learning.

Owen, T. M., Fisher, W. W., Akers, J. S., Sullivan, W. E., Falcomata, T. S., Freer, B. D., et al. (2020). Treating destructive behavior reinforced by increased caregiver compliance with the participant's mands. Journal of Applied Behavior Analysis, 53, 1494-1513.

Rossetti, L. M. (2006). The Rossetti infant-toddler language scale. Dallas, TX: Linguisystems.

Schmidt, J. D., Bednar, M. K., Willse, L. V., Goetzel, A. L., Concepcion, A., Pincus, S. M., et al. (2017). Evaluating treatments for functionally equivalent problem behavior maintained by adult compliance with mands during interactive play. Journal of Behavioral Education, 26, 169-187. https://doi.org/10.1007/s10864-0169264-1.

Torres-Viso, M., Strohmeier, C. W., \& Zarcone, J. R. (2018). Functional analysis and treatment of problem behavior related to mands for rearrangement. Journal of Applied Behavior Analysis, 51, 158165. https://doi.org/10.1002/jaba.437.

Implications for Practice - This Brief Practice provides a framework to conceptualize assessment of challenging behavior maintained by access to adult compliance with nonvocal mands. 
- This study replicates prior work in the area and extends the research by documenting effects for a nonvocal individual with challenging behavior maintained by access to adult compliance with mands. It provides a possible method for conducting schedule thinning within a multicomponent treatment package.

- Providers should carefully examine the relationship between communication and challenging behavior while conducting functional behavior assessments and consider both vocal and nonvocal communication.

Publisher's Note Springer Nature remains neutral with regard to jurisdictional claims in published maps and institutional affiliations. 